\title{
Desenvolvimento das Políticas e dos Cuidados de Saúde Sexual e Reprodutiva em Portugal
}

\author{
Maria José Santos \\ Elisabete Ferreira \\ Anabela Figueiredo \\ Manuela Ferreira
}

\begin{abstract}
Resumo
O entendimento sobre o conceito de saúde sexual e reprodutiva (SSR) é relativamente recente e tem conhecido transformações substanciais ao longo do tempo, resultado de um conjunto de fatores socioculturais, políticos e médico-cientificos, que permitiram reconhecer que os indivíduos têm o direito a desfrutar da sexualidade de uma forma livre e esclarecida. As mudanças no contexto histórico, social e cultural que atravessaram todo o século XIX e XX influenciaram claramente a SSR em Portugal. Mudanças que foram mais significativas na segunda metade do século XX, observando-se nas décadas de 1960 e 1970 a promoção da contraceção e planeamento familiar, a legislação sobre sexualidade juvenil e o acesso dos jovens às consultas de planeamento familiar. Na década de 1980 e seguintes, a generalização do acesso à contraceção e os debates sobre a sexualidade juvenil e políticas de educação sexual. Nestas décadas, surgem novas preocupações relacionadas com pandemia do VIH/SIDA, que obrigam a equacionar os comportamentos sexuais como fator de risco para a saúde. Entre 2000 e 2010, assiste-se à integração da educação sexual em todos os níveis de ensino, com exceção do pré-escolar, numa aceção verdadeiramente democrática da educação sexual. Mais recentemente, realidades emergentes como a democratização do uso da internet e das redes sociais, levantam novos desafios e novos riscos para a saúde que não devem ser negligenciados. Com este artigo pretendemos descrever e analisar o desenvolvimento político-social e dos cuidados na SSR em Portugal, como garantia do direito à informação e a serviços de saúde de qualidade, acessiveis a todos os cidadãos.
\end{abstract}

Palavras-chave: Cuidados de saúde; Políticas; Saúde Sexual e Reprodutiva.

\begin{abstract}
The understanding of the concept of sexual and reproductive health $(\mathrm{SRH})$ is relatively recent and has undergone substantial transformations over time, as a result of a set of sociocultural, political and medical-scientific factors that have allowed the recognition that individuals have the right to enjoy of sexuality in a free and enlightened way. Changes in historical, social and cultural context that crossed all the nineteenth and twentieth century clearly influenced the SRH in Portugal. Changes that were most significant in the second half of the twentieth century saw the promotion of contraception and family planning and legislation on youth sexuality in the 1960s and 1970s. In the 1980 s and beyond, the generalization of access to contraception and debates on youth sexuality and sexual education policies In recent decades, new concerns about the HIVIAIDS pandemic have arisen, and there is a need to address risky sexual behavior. Between 2000 and 2010, the integration of sex education at all levels of education, with the exception of preschool, in a truly democratic sense of sex education is observed. More recently, emerging realities such as the democratization of the use of the Internet and media, pose new challenges and new risks to health that should not be neglected. With this article we intend to describe and analyze the socio-political development and care in the SRH in Portugal, as a guarantee of the right to information and quality health services, accessible to all citizens.
\end{abstract}

Keywords: Care Health; Policies; Sexual and Reproductive Health.

\section{INTRODUÇÃO}


Na maioria dos países, a saúde sexual e reprodutiva (SSR) emergiu como uma área prioritária a considerar nas políticas e programas de saúde, revelando uma preocupação com os potenciais problemas de saúde que podem afetar, direta ou indiretamente, o bem-estar dos indivíduos e das comunidades, com custos elevados ao nível do capital humano e da produtividade ${ }^{1}$. 0 entendimento sobre os conceitos de saúde reprodutiva e saúde sexual são relativamente recentes e têm conhecido transformações substanciais ao longo do tempo, em resultado de um conjunto de fatores socioculturais, políticos e médicocientíficos, que permitiram reconhecer que os indivíduos têm o direito a desfrutar da sexualidade de uma forma livre, segura e esclarecida. A queda generalizada da fecundidade no mundo facilitou a mudança de paradigma do planeamento familiar para os direitos sexuais e reprodutivos. Estes passam a ser considerados direitos individuais, independentemente da idade, género, orientação sexual, religião ou etnia e abrangem a sexualidade e a fecundidade, o direito à informação e o acesso aos serviços de saúde, através de uma abordagem interdisciplinar e não apenas biomédica².

$\mathrm{Na}$ abordagem das políticas de SSR em Portugal, devemos considerar diferentes fases que refletem alguns dos factos mais relevantes a nível nacional e internacional, que permitiram 0 desenvolvimento das políticas de SSR. Com este artigo pretendemos descrever e analisar 0 desenvolvimento político-social e dos cuidados na SSR em Portugal, como garantia do direito à informação e a serviços de saúde de qualidade, acessíveis a todos os cidadãos.

\section{DO CRESCIMENTO DEMOGRÁFICO ÀS POLÍTICAS DE CONTROLO DE NATALIDADE}

As mudanças no contexto histórico, social e cultural que atravessaram todo o século XIX e em particular a segunda metade do século XX influenciaram as políticas de saúde reprodutiva em todo 0 mundo e também em Portugal. A discussão sobre as questões relacionadas com a reprodução e natalidade surgiram com Thomas Malthus (1766-1834), economista e pastor da igreja Anglicana, considerado o pai da demografia, que preocupado com as reservas do mundo, publicou em 1798 "Um Ensaio Sobre o Principio da População", onde apresentou uma posição pessimista e conservadora relativa às políticas de natalidade, uma vez que não separava a atividade sexual da reprodução. Malthus argumentava que a população tendia sempre a crescer mais rapidamente do que os meios de subsistência, pelo que o crescimento e o progresso seriam utópicos e não estariam ao alcance da maioria

\footnotetext{
${ }^{1}$ Fundo das Nações Unidas Para a População. Situação da população mundial 2005: A promessa de igualdade: equidade em matéria de género, saúde reprodutiva e objectivos de desenvolvimento do milénio. (FNUAPA, 2005) http://www.unric.org/pt/actualidade/6259 (acesso em 24 de abril 2017).

2 Duarte Vilar. Contributos para a história das políticas de saúde sexual reprodutiva em Portugal

(Lisboa: Centro de Estudos Sociais da Universidade de Coimbra, 2009), 28.
} 
da população ${ }^{3}$. Foi Francis Place (1822), quem considerou pela primeira vez, que a resposta aos problemas populacionais poderia residir no uso de métodos contracetivos artificiais e fundou a primeira organização de controlo da natalidade. À problemática levantada por Malthus relativa à explosão demográfica veio juntar-se, no século XIX, um conjunto de fatores sociais, tais como o desenvolvimento da sociedade industrial, a entrada da mulher no mundo do trabalho e o avanço do conhecimento médico, que permitiram uma diminuição significativa das taxas de mortalidade infantil e o aumento da esperança média de vida, conduzindo a um aumento populacional que criou a necessidade de implementar políticas de controlo da natalidade 4 .

No início do século XX, em diversos países da Europa e nos Estados Unidos da América, multiplicaram-se atividades pioneiras no domínio da maternidade livremente assumida e do controlo da natalidade. Nos EUA, Margaret Sanger, enfermeira, educadora sexual e ativista, foi a primeira mulher a popularizar o termo "birth control" e a abrir a primeira clínica de controle de natalidade nos EUA (1916) e outros estabelecimentos ligados à organização Planned Parenthood Federation of America. Em 1914, Margaret Sanger foi processada pelos EUA, pois à data era proibida a circulação, venda e produção de toda literatura com conteúdos de carácter sexual, erótico ou com informações sobre controlo de natalidade ${ }^{5}$.

Nos anos 20 foi fundada a Liga Internacional com o objetivo de difundir este movimento e promover a realização de Conferências Internacionais da População. Depois da contraceção e regulação da natalidade, apenas nos anos 30, se chega ao conceito de planeamento familiar, que antecedeu 0 conceito de saúde reprodutiva. Esta designação encerra dois significados destintos, por um lado, a separação do sexo da reprodução pelo uso de métodos contracetivos e, por outro lado, um esforço no controlo do crescimento populacional, como medida de combate ao subdesenvolvimento social e económico ${ }^{6}$. Nas décadas de 1950-1960, observou-se o maior crescimento demográfico de toda a história da humanidade, difundindo-se o medo da "explosão populacional". Foi neste ambiente de alto crescimento demográfico e sob influência do pensamento neomalthusiano, que ocorreram as primeiras Conferências Internacionais de População e Desenvolvimento organizadas pela Organização das Nações Unidas

\footnotetext{
${ }^{3}$ Silva, Wellington Souza. Teoria populacional Malthusiana. (Centro Universitário Fundação Santo André, 2014), https://www.infoescola.com/geografia/teoria-populacional-malthusiana/ (acessado em 12 de maio de 2019).

${ }^{4}$ Helena, I. Manuel. "Conhecimentos, atitudes e práticas sobre planeamento familiar de mulheres timorenses residentes em Portugal" (dissertação de mestrado, Unversidade Aberta, 2007) 237.

${ }^{5}$ Vicki Cox, Margaret Sanger - Rebel for Women's Right (EUA: Chelsea House Publishers, 2004) 3-7.

${ }^{6}$ Ibid.
} 
(ONU), iniciando-se o debate sobre os direitos humanos, bem-estar social, igualdade de género e, em especial, sobre a saúde e direitos reprodutivos ${ }^{7}$.

Na segunda metade do século XX a saúde e o bem-estar passaram a ser considerados direitos dos indivíduos, aceitando-se que um elevado número de filhos poderia ser prejudicial para a saúde das mulheres e dos recém-nascidos, o que resultou na afirmação da necessidade de espaçamento entre as gravidezes, aceitando-se os métodos contracetivos não só como medida de controlo da natalidade, mas também como forma efetiva de planear os nascimentos de acordo com os desejos de cada família. Quando as pesquisas demográficas passaram a mostrar que o número desejado de filhos, por parte das mulheres e casais, era menor que a fecundidade, passou a dar-se importância às necessidades não atendidas de contraceção. Em 1952, foi fundada, na conferência de Bombaim, a International Planned Parenthood Federation (IPPF), proclamando-se que o planeamento familiar é um direito humano fundamental, mas só em 1968 a Organização Mundial da Saúde (OMS) reconhece o planeamento familiar como componente fundamental dos serviços de saúde ${ }^{8}$. A nível internacional, os movimentos de apoio ao planeamento familiar estavam em franco desenvolvimento procurando legalizar e afirmar 0 acesso à contraceção que, à semelhança de Portugal, era ilegal em países como os EUA ou a França. Em 1960, nos EUA foi aprovada a venda da Enovid-10®, a primeira pílula anticoncecional, que deu inicio à revolução sexual no mundo ocidental. Esta pílula foi desenvolvida pelo cientista Gregory Pincus, aparentemente a pedido Margaret Sanger e da milionária Katherine McCormick, que apoiaram a investigação para 0 desenvolvimento de uma pílula contra a gravidez que fosse fácil de usar, eficiente e barata. Esta pílula foi desenvolvida durante cinco anos como um medicamento para aliviar os sintomas da menstruação, pois os contracetivos estavam oficialmente proibidos nos EUA até $1965^{9}$.

Em Portugal, nos anos 60, o planeamento familiar era ainda considerado subversivo e semiclandestino, aceitando-se apenas os métodos naturais propostos pela igreja católica, como o coito interrompido e as irrigações vaginais, apesar do preservativo e a pílula já serem comercializados em Portugal desde 1962, mas a pílula contracetiva era usada apenas com indicação terapêutica para regular o ciclo menstrual, pois era considerada um método abortivo e o seu uso punível por lei. 0 controlo da fecundidade era feito sobretudo pelos homens através do coito interrompido e do preservativo ${ }^{10}$.

\footnotetext{
7 Sónia Corrêa, José E. D. Alves \& Paulo M. Jannuzzi, Direitos e saúde sexual e reprodutiva: Marco teórico conceitual e sistema de indicadores. Indicadores municipais de saúde sexual e reprodutiva (Rio de Janeiro: ABEP, 2006), 62.

${ }^{8}$ Bebiana Gomes, "Para a história do planeamento familiar", Revista da Associação para o Planeamento Familiar 37 (1988): 2-5.

9 Hartl, Judith. 1960: Primeira pílula anticoncepcional chega ao Mercado. (2018) https://p.dw.com/p/2zoqhttps (acessado em 4 de maio de 2019).

${ }^{10}$ Angus, Mclaren, História da Contracepção - da Antiguidade à Actualidade (1990). (reimpressão, Lisboa: Terramar,1997), 116-117.
} 


\section{DO PLANEAMENTO FAMILIAR AOS CUIDADOS EM SAÚDE SEXUAL E REPRODUTIVA}

$O$ início do planeamento familiar em Portugal tem como referência a criação da Associação Planeamento Familiar (APF), em 1967, que permitiu que um conjunto de profissionais de saúde, dedicados à prestação de cuidados da família, iniciasse o debate sobre a necessidade de implementação do planeamento familiar em Portugal. A partir dos anos 70, o direito de escolha e a liberdade de decisão nos assuntos sexuais e reprodutivos tornam-se aspetos inegociáveis nas agendas políticas dos diferentes países, ganhando relevância as questões relativas à saúde integral da mulher e à SSR, incluindo o acesso à contraceção e ao aborto seguro ${ }^{11}$.

Após a Revolução de 25 de abril, em 1974, um texto oficial do Programa de Ação do Ministério dos Assuntos Sociais, fez a primeira referência expressa à necessidade de se informar os cidadãos sobre o planeamento familiar. A elevada taxa de mortalidade infantil $(59,2 / 1.000)$ e materna $(84,6 / 100.000$, quase uma mulher em cada mil), sobretudo como resultado de complicações por aborto clandestino terão justificado as medidas que se seguiram. Em 1976, o Dr. Albino Aroso, então secretário de estado da saúde, considerado o pai do planeamento familiar em Portugal, determinou a criação de consultas de planeamento familiar em todos os centros de saúde do país, integradas nos serviços de saúde materna e infantil. Nesta perspetiva, a constituição portuguesa é uma das raras no mundo a consignar, já em 1976, 0 direito dos cidadãos ao planeamento familiar (artigo 67\%), apontando como deveres do Estado, assegurar 0 direito ao planeamento familiar. $O$ processo de implementação das consultas de planeamento familiar foi lento e só no final dos anos 70 estas consultas estavam disponíveis nos centros de saúde. 0 controlo da conceção iniciou um percurso mais consistente a partir de 1974 e, apesar das assimetrias existentes, a história da contraceção em Portugal foi pautada por algum sucesso. No início da década de 1980, encontrava-se em funcionamento cerca de 400 consultas de planeamento familiar nos centros de saúde e hospitais. Apesar dos avanços legislativos, o planeamento familiar continuava a ser desconhecido e pouco usado por uma grande parte da população, e as políticas de saúde reprodutiva centravam-se quase exclusivamente na promoção do acesso à contraceção e educação contracetiva das mulheres, sem envolvimento dos homens.

A década de 1980 foi o momento de viragem nas questões específicas da saúde dos adolescentes e jovens. Iniciaram-se múltiplas experiências de promoção da saúde, atendimento, investigação, formação e planeamento e organização de cuidados de saúde reprodutiva adaptados a este grupo. Em termos legislativos, foram marcos essenciais na afirmação da SSR dos jovens em Portugal, a lei $n^{0} 3 / 84$, de 24 de março ${ }^{12}$, que estabelece o direito e à educação sexual e define as formas de acesso

\footnotetext{
11 Ibd., 4.

12 Lei n. ${ }^{0}$ 3/84, de 24 de março. Estabelece o direito à educação sexual e define as formas de acesso ao planeamento familiar. Diário da República, 71. Série I.
} 
ao planeamento familiar, a lei $n^{0} 4 / 84$, de 5 de abrili3, que regula a proteção e defesa da maternidade e paternidade, a lei $n^{0} 6 / 84$, de 11 de maio ${ }^{14}$, que estabelece a exclusão de ilicitude de alguns casos de interrupção voluntária da gravidez e a portaria $n^{0} 52 / 85$, de 26 de janeiro ${ }^{15}$, que regula o funcionamento das consultas de planeamento familiar e centros de atendimento a jovens. Estas leis vieram garantir 0 desenvolvimento das consultas de planeamento familiar e dos Centros de Atendimento de Jovens (CAJ), assegurando 0 acesso sem restrições às consultas de planeamento familiar a todos os indivíduos e, em particular, a todos os jovens em idade fértil. Embora com dezenas de anos de atraso em relação à legislação dos países europeus, Portugal passa a dispor dos instrumentos legais para que a parentalidade não seja fruto do acaso, mas uma escolha livre, consciente e responsável16.

Foi também no início dos anos 80 que a OMS começou a utilizar o conceito de saúde sexual, transpondo o tradicional conceito de saúde para a área da vivência da sexualidade ${ }^{17}$. Na construção deste conceito, a pandemia do VIH/SIDA teve um papel importante, uma vez que houve necessidade de equacionar a saúde reprodutiva não apenas centrado na saúde materna, mas também nas questões da saúde sexual, com ênfase particular na prevenção do VIH/SIDA e nos comportamentos sexuais de risco. $\mathrm{O}$ combate à infeção pelo VIH/SIDA foi assumido como um objetivo prioritário, implementando-se inúmeras campanhas de prevenção da infeção e promoção do uso do preservativo. Esta nova abordagem permitiu a definição de políticas públicas de saúde focadas na diversidade de comportamentos sexuais, permitindo um avanço significativo nas questões da sexualidade ${ }^{18}$. Os comportamentos sexuais passaram a ser considerados uma questão de urgência social e epidemiológica, como um fator que pode influenciar diretamente a saúde dos indivíduos e determinar as políticas de saúde pública ${ }^{19}$.

A década de 1980 centra-se, sobretudo, na generalização do acesso à contraceção, nos debates sobre a sexualidade juvenil e das políticas de educação sexual, tendo como dinamizadores o parlamento e as organizações não-governamentais que se posicionam de forma mais liberal ou mais conservadora sobre as políticas relacionadas com a sexualidade juvenil. O Ministério da Educação começa a ter um

\footnotetext{
${ }^{13}$ Lei n. 0 4/84, de 5 de abril. Proteção da maternidade e paternidade. Diário da República, 81. Série I.

14 Lei n. ${ }^{\circ} / 84$, de 11 de maio. Exclusão de ilicitude em alguns casos de interrupção voluntária da gravidez. Diário da República, 109. Série I.

${ }^{15}$ Portaria no 52/85, de 26 de janeiro. Regulamento das consultas de planeamento familiar e centros de atendimento para jovens. Diário da República, 22. Série I.

${ }^{16}$ Bebiana. S. Gomes, "Para a história do planeamento familiar", Revista da Associação para o Planeamento Familiar 37 (1988): 2-5.

17 Duarte Vilar, "O estado das coisas". Revista da Associação para o Planeamento Familiar 36 (1986):8-16.

18 Duarte Vilar, Falar disso (Porto: Afrontamento, 2003).

19 Pedro Moura. Ferreira, \& Manuel. V. Cabral, Sexualidades em Portugal: Comportamentos de risco (Lisboa: Editorial Bizâncio, 2010) 487.
} 
papel central na educação sexual, promovendo, no contexto da educação para a saúde, ações e programas de educação sexual20.

\section{DOS DIREITOS SEXUAIS, AO CONCEITO UNIVERSAL DE SAÚDE SEXUAL E REPRODUTIVA}

A década de 90 é marcada por dois acontecimentos a nível internacional, com reflexos significativos na evolução das políticas e direitos de SSR em todo o mundo: a aprovação da Carta dos Direitos Sexuais e Reprodutivos pela IPPF e a consagração dos direitos reprodutivos com delineamento de políticas de saúde a nível mundial, resultado da Conferência Internacional sobre População e Desenvolvimento, realizada no Cairo em 1994 e, posteriormente, em 1995, a Conferência Internacional Sobre a Mulher, realizada em Pequim ${ }^{21}$. Os direitos sexuais passam a ser direitos humanos reconhecidos internacionalmente e que incluem vários direitos humanos fundamentais, tais como: o direito à privacidade, o direito à saúde, o direito à liberdade de pensamento e expressão, o direito de viver livre de violência e 0 direito à educação e informação22.

A Conferência do Cairo foi um dos marcos mais significativos na redefinição de políticas de SSR, ao denunciar práticas coercivas, ao iniciar a discussão sobre temas polémicos como o aborto clandestino e a considerar a relação entre população e desenvolvimento. Observou-se, desde então, uma mudança na abordagem nos temas da demografia, com as perspetivas de controlo populacional a perder força a favor do planeamento familiar, pautado pelos direitos reprodutivos, como uma das ferramentas fundamentais de promoção da SSR, suportados por políticas sociais e económicas para reforçar os direitos das mulheres ${ }^{23}$. Desde então reconhecesse à mulher o direito de regular a sua própria fecundidade, direito que deve ser garantido por programas e políticas de planeamento familiar, destacam-se as condições que os serviços de saúde devem garantir, para que as mulheres possam ter uma gravidez vigiada e um parto seguro. Estes direitos são reafirmados na Conferência de Pequim, salientando-se que a falta de atenção aos direitos reprodutivos limita as oportunidades da mulher na vida pública e privada, bem como 0 acesso à educação e ao exercício de outros direitos sociais e políticos ${ }^{24}$.

\footnotetext{
20 Ibid., 2.

${ }^{21}$ Viotti, Maria Luiza. Declaração e Plataforma de Ação da IV Conferência Mundial sobre a Mulher (Pequim,1995), http://www.onumulheres.org.br/wp-content/uploads/2014/02/declaracao pequim.pdf (acessado em 04 de maio de 2019).

${ }^{22}$ International Planned Parenthood Federation, Exclaim - young people's guide to 'Sexual rights: an IPPF declaration (London: IPFF, 2011), 40.

${ }^{23}$ Santos, A. E., Leão, F. M., Araújo, L. S., \& Ferreira, L.T. Saúde sexual e reprodutiva: Direitos e desafios em um mundo multicultural. (2011) http://www.sinus.org.br/2011/press/downloads/oms.pdf (acessado em 02 de maio de 2019).

${ }^{24}$ Maria H. Pimentel, Leonel Preto, Maria J. Alves, \& Ana M. Monteiro, "Comportamento sexual e estudantes do ensino superior", Psicologia, Saúde \& Doenças, 17,3 (2016):352-367.

http://dx.doi.org/10.15309/16psd170304 (acessado em 04 de junho de 2019).
} 
O Programa de Ação do Cairo, apontou ainda para a necessidade de se abordar a SSR dos jovens de uma forma integral, centrada na pessoa e no respeito pelo outro, com o objetivo de reduzir a gravidez adolescente e os comportamentos de riscos associados às infeções sexualmente transmissíveis (IST) e de promover a igualdade de género na definição de políticas de SSR.

Foi no decurso destas conferências internacionais que o conceito de saúde reprodutiva foi ampliado e redefinido como sendo: "...um estado de completo bem-estar físico, mental e social, e não apenas a ausência de doença ou enfermidades, em tudo o que se relaciona com o sistema reprodutivo, às suas funções e processos"25.

Desta forma, a saúde reprodutiva constitui-se como parte integrante do conceito universal de saúde da OMS, e implícito na sua definição está o direito dos indivíduos à informação, o acesso aos métodos contracetivos e ao planeamento familiar eficazes, seguros e financeiramente compatíveis com a sua situação económica. Assim como o acesso a serviços e cuidados de saúde apropriados que capacitem as mulheres para a gravidez e parto, e que proporcionem aos casais as melhores oportunidades de terem filhos saudáveis.

O conceito de saúde sexual surge mais tarde como resultado de um Convénio Internacional de Saúde Sexual, promovido pela OMS em 2002. A saúde sexual passa a ser definida pela OMS²6 como "um estado de completo bem-estar físico, emocional, mental e social associado à sexualidade e não só a ausência de doença ou enfermidade". Esta definição mais abrangente e positiva, reforça a noção que a saúde sexual deve pressupor, a existência de "experiências prazerosas e sexo seguro, livre de coerção, discriminação e violência. Para se alcançar e manter a saúde sexual, os direitos sexuais de todas as pessoas devem ser respeitados, protegidos e satisfeitos"27, não se restringindo apenas à prevenção de disfunções sexuais ou IST. Nesta perpectiva podemos considerar que o conceito de saúde sexual é parte integrante do conceito de saúde reprodutiva, que visa potenciar a vida, as relações pessoais e o bem-estar geral, dado que, nas sociedades contemporâneas, a sexualidade não está limitada apenas à reprodução 28 . Os conceitos de saúde sexual e de saúde reprodutiva tornam-se mais abrangentes, ultrapassando a dimensão médica e preventiva a que tradicionalmente as questões de saúde aparecem associadas. Passam a considerar os comportamentos individuais, as relações de intimidade, a proteção da saúde

\footnotetext{
25 International Planned Parenthood Federation, Reproductive Health (IPPF, http://www.ippf.org/en/Resources/Glossary.htm?g=R.

${ }^{26}$ World Health Organization. Defining Sexual Health. Report of a technical consultation on sexual health (WHO, 2006)

https://www.who.int/reproductivehealth/publications/sexual health/defining sexual health.pdf?ua=1 27 Ibid.

28 World Health Organization, The sexual and reproductive health of younger adolescents: Research issues in developing countries (WHO, 2011), http://apps.who.int/iris/bitstream/10665/44590/1/9789241501552 eng.pdf (acessado em 23 de maio 2018).
} 
física e mental, os direitos individuais e sociais e o direito à informação e acesso aos serviços e cuidados de saúde ${ }^{29}$. No mesmo sentido, o conceito de SSR passa a ser amplamente utilizado, uma vez que os dois conceitos - saúde sexual e saúde reprodutiva - se complementam numa abordagem integrada da sexualidade humana.

Beneficiando da conjuntura internacional, no final dos anos 90 é aprovada em Portugal a lei $n^{0}$ 120/99, de 11 de agosto, que vem a ser regulamentada pelo decreto-lei $n^{0}$ 259/2000, de 17 de outubro30, integrando já o novo conceito de SSR, que veio reforçar as garantias do direito dos indivíduos à saúde reprodutiva, abordando temas como a promoção da educação sexual e a saúde reprodutiva, a prevenção de IST, métodos contracetivos, planeamento familiar e interrupção voluntária da gravidez (IVG). A lei $n^{\circ}$ 12/2001 de 29 de maio31, vem regulamentar a distribuição gratuita de contracetivos, incluindo a contraceção de emergência, em todas as unidades de saúde da rede de cuidados de saúde primários e hospitalares.

\section{DOS DIREITOS SEXUAIS AO CONCEITO UNIVERSAL DE SAÚDE SEXUAL E REPRODUTIVA}

Entre 2000 e 2002, assiste-se ao desenvolvimento da educação sexual nas escolas, impulsionado pelo decreto-lei $n^{0}$ 259/2000, de 17 de outubro ${ }^{32}$, que estabeleceu que cada escola deveria integrar a educação sexual no seu projeto educativo. Este decreto-lei veio contemplar a implementação de programas de promoção da educação sexual na escola, adequados aos diferentes grupos etários, com envolvimento da comunidade educativa, serviços de saúde da área, associações de estudantes e de pais/encarregados de educação. Preconiza, ainda, a prevenção das IST, considerando a importância da utilização do preservativo na prevenção das mesmas e a disponibilização do preservativo através de meios mecânicos a todos os estabelecimentos de ensino secundário e superior, por decisão dos conselhos diretivos, ouvidas as associações de pais e estudantes. Prevê também o acesso dos jovens a consultas de planeamento familiar, nos centros de saúde ou a nível hospitalar, mesmo que fora da área de residência, bem como a criação de consultas de planeamento familiar integradas nos serviços de saúde, nos estabelecimentos de ensino superior, com o objetivo de aconselhar e dar apoio técnico na utilização dos métodos contracetivos.

\footnotetext{
29 Ibden., 2.

30 Decreto-Lei no 259/2000, de 17 de outubro. Regulamenta a Lei n.o 120/99, de 11 de Agosto (reforça as garantias do direito à saúde reprodutiva), fixando condições de promoção da educação sexual e de acesso dos jovens a cuidados de saúde no âmbito da sexualidade e do planeamento familiar. Diário da República, 240. Série I-A.

31 Lei no 12/2001, de 29 de maio. Contraceção de emergência. Diário da República, 124. Série I-A.

32 Decreto-Lei no 259/2000, de 17 de outubro. Regulamenta a Lei n.o 120/99, de 11 de Agosto (reforça as garantias do direito à saúde reprodutiva), fixando condições de promoção da educação sexual e de acesso dos jovens a cuidados de saúde no âmbito da sexualidade e do planeamento familiar. Diário da República, 240. Série I-A.
} 
No âmbito da educação sexual, seguiram-se outros os marcos legislativos importantes, como foi a publicação do despacho $n^{0}$ 19737/2005, de 13 de Setembro ${ }^{33}$, que determinou a criação de uma comissão independente - Grupo de Trabalho em Educação Sexual: GTES - para avaliar os conteúdos dos programas de educação sexual e propor os parâmetros gerais de educação sexual em meio escolar, numa perspetiva de promoção da saúde dos jovens. No seu relatório final, apresentado em 2007, este grupo de trabalho recomendou que a abordagem da saúde sexual considerasse o entendimento da sexualidade como uma das componentes da pessoa, no contexto de um projeto de vida que englobasse valores e uma dimensão ética, a compreensão dos aspetos relacionados com as principais IST (incluindo o VIH/SIDA), a maternidade na adolescência, a IVG e o uso de métodos contracetivos, em particular o preservativo para a prevenção da gravidez e das $I S T^{34}$. A obrigatoriedade da educação sexual nas escolas já prevista desde 1999 (lei $n^{0}$ 120/99), foi reforçada pela lei $n^{0}$ 60/2009, de 6 de agosto ${ }^{35}$, que estableceu o regime da educação sexual em meio escolar, em todos os níveis de ensino, com exceção do ensino pré-escolar. $A$ portaria $n^{0}$ 196-A/2010, de 4 de abril36, procede à sua regulamentação, estabelecendo a obrigatoriedade de educação sexual em todas as instituições de ensino básico e secundário, público e privado, a nível nacional, numa aceção verdadeiramente democrática ${ }^{37}$. A lei prevê, ainda, que a educação sexual tenha 0 acompanhamento de profissionais de saúde e a possibilidade de serem estabelecidas parceria com entidades credenciadas na área da educação para a saúde e educação sexual para o desenvolvimento de projetos específicos, através das unidades de saúde pública locais. 0 processo de implementação da educação sexual nas escolas deve ser continuamente monitorizado, uma vez que é reconhecida a sua importância, pois a educação sexual é fundamental no controlo da fertilidade e na prevenção das IST, com consequências positivas na sexualidade, gravidez, fertilidade, vigilância pré-concecional e pré-natal, qualidade e sobrevivência das crianças $^{38}$. Apesar do quadro legislativo atual as dificuldades na implementação da educação sexual nas escolas mantém-se, pois embora a educação sexual tenha sido

\footnotetext{
${ }^{33}$ Despacho no 19737/2005, de 13 de setembro. Cria um grupo de trabalho no âmbito do Ministério da Educação com o objetivo de estudar e propor os parâmetros gerais dos programas de educação sexual em meio escolar, na perspetiva da promoção da saúde escolar. Diário da República,176. Série 2.

${ }^{34}$ Lúcia Ramiro, Marta Reis, Margarida G. Matos, José Diniz, \& Celeste Simões, "Educação sexual, conhecimentos, crenças, atitudes e comportamentos nos adolescentes", Revista Portuguesa de Saúde Pública, 29,1 (2011):11-21.

${ }^{35}$ Lei no 60/2009, de 6 de agosto. Estabelece o regime da aplicação da educação sexual em meio escolar. Diário da República, 151. Série I.

${ }^{36}$ Portaria no 196-A/2010, de 4 de abril. Regulamenta a Lei n. ${ }^{0}$ 60/2009, de 6 de Agosto, que estabelece o regime de aplicação da educação sexual em meio escolar

37 Margarida G. Matos, Marta Reis, Lúcia Ramiro, José Pais-Ribeiro \& Isabel Leal. "Educação sexual em Portugal: Legislação e avaliação da implementação nas escolas", Psicologia, Saúde \& Doenças, 15,2 (2014):335-355.

38 Direção-Geral da Saúde, Saúde Reprodutiva. Planeamento familiar (Lisboa: DGS, 2008), 69.
} 
considerada obrigatória há mais de uma década, em 2015, apenas $67,4 \%$ dos jovens refere ter acesso a educação sexual/informação sobre contraceção e prevenção de IST ${ }^{39}$.

Mais recentemente, realidades emergentes como, por exemplo, a democratização do uso da internet e as redes sociais, levantam novos desafios e preocupações nesta área. As novas tecnologias de informação criam um ambiente extremamente apelativo e com novas oportunidades de vivência da sexualidade, mas também com novos riscos que devem ser antecipados ${ }^{40}$. De facto o mundo digital pode representar um risco para a saúde quando se extrapola os limites entre o real e o virtual, entre o público e o privado, entre o que é informação ou exploração, entre a intimidade e a distorção dos factos ou imagens "reais". Para prevenir os problemas e ameaças que se colocam à saúde sexual desta geração digital é necessário que os profissionais de saúde reconheçam a necessidade de se manterem atualizados. Termos como cyberbullying, grooming, sexting, messaginge texting, fazem parte do quotidiano dos jovens e também devem fazer parte desta atualização, por parte dos profissionais de saúde 41 .

\section{CONSIDERAÇõES FINAIS}

O desenvolvimento de políticas de SSR em Portugal foi marcado, na segunda metade do século XX, por algum sucesso, e foi acompanhando os intensos debates morais e políticos, nomeadamente a promoção da contraceção e planeamento familiar nas décadas de 60 e 70, a legislação sobre sexualidade juvenil e 0 acesso dos jovens às consultas de planeamento familiar, nos anos 80 e seguintes, a formalização da contraceção de emergência e o aborto legal e seguro, que percorre todo este período e, mais recentemente, a implementação da educação sexual nas escolas. É incontornável que todo o quadro legislativo que surgiu nas décadas de 1980/90 foi de extrema importância para o desenvolvimento das políticas de SSR em Portugal, permitindo que, no início deste século, estivessem reunidas as condições para uma efetiva aplicação das orientações constantes da legislação. Diversas questões de SSR, como a sexualidade juvenil, a educação sexual, o acesso à contraceção e a IVG, foram alvo de novos enquadramentos legais e novas políticas no contexto da educação, juventude e saúde, e foi neste confronto ideológico e político que se foram produzindo e implementando as políticas de SSR. Os resultados alcançados nestas quatro décadas são claramente positivos, pelos direitos alcançados, pelos ganhos de saúde, pela autonomia e empoderamento das mulheres, sobretudo através do acesso à

\footnotetext{
39 Margarida G. Matos, Celeste Simões, Isabel Camacho, Marta Reis \& Equipa Aventura Social, A saúde dos adolescentes portugueses em tempos de recessão, dados nacionais 2014. (Lisboa: Centro de Malária e Outras Doenças Tropicais /IHMT/UNL,FMH/ Universidade de Lisboa. 2015) 195.

40 Associação para o Planeamento da Família. Para um programa nacional de saúde sexual e reprodutiva. (Lisboa: APF, 2015), http://www.apf (acessado 04 de maio de 2017).

${ }^{41}$ Evelyn Eisenstein, "Desenvolvimento da sexualidade da geração digital", Adolescência \& Saúde, 10,1 (2013): 61-71.
} 
contraceção e às consultas de planeamento familiar como instrumento de controlo da fecundidade e por inerência pelo controlo do seu próprio percurso de vida ${ }^{42}$.

É incontestável que, nos últimos anos, Portugal registou melhorias significativas dos indicadores relativos à SSR, nomeadamente: i) na utilização dos métodos contracetivos, incluindo dos métodos menos dependentes, ou não dependentes da utilizadora ${ }^{43}$, iii) na diminuição progressiva do número de interrupções de gravidez por opção da mulher ${ }^{44}$, e iv) na diminuição de complicações maternas por aborto inseguro45. Contudo, as modificações sociais que se têm registado nos últimos anos, a par das dificuldades económicas e da necessidade de contenção das despesas no SNS, colocam desafios acrescidos que devem ser equacionados de forma a não comprometer os ganhos em saúde já alcançados $^{46}$. São as leis e políticas em vigor que protegem os indivíduos mais vulneráveis, garantindo 0 direito à informação e serviços de saúde de qualidade, acessíveis, de uma forma equitativa a todos os cidadãos ${ }^{47}$. Pelo exposto, é nosso entendimento que a evolução das políticas de SSR só será possível se existir a crença fundamental na sociedade de que a SSR é parte integrante da saúde e bem-estar, um direito fundamental de todos os indivíduos ao longo do ciclo de vida e uma garantia para a autonomia e desenvolvimento social.

\footnotetext{
42 Ibid., 2.

43 Fernanda Águas, Teresa Bombas \& Daniel Silva, "Avaliação das práticas contracetivas das mulheres em Portugal", Acta Obstétrica e Ginecológica Portuguesa, 10, 3 (2016):1-9.

44 Divisão de Saúde Sexual Reprodutiva Infantil e Juvenil, Relatório dos registos das interrupções da gravidez dados de 2015 (Lisboa: DGS, 2016) 38.

45 Divisão de Saúde Sexual Reprodutiva Infantil e Juvenil, Relatório de análise das complicações relacionadas com a interrupção da gravidez 2013 - 2014 (Lisboa: DGS, 2015).

${ }^{46}$ Divisão de Saúde Reprodutiva, Compromisso com os princípios enunciados nos objectivos para o desenvolvimento do milénio relativos à saúde materno-infantil (Lisboa: DGS, 2010) 4.

${ }^{47}$ World Health Organization, The sexual and reproductive health of younger adolescents: Research issues in developing countries (WHO, 2011), http://apps.who.int/iris/bitstream/10665/44590/1/9789241501552 eng.pdf (acessado em 23 de maio 2018).
} 


\section{SOBRE OS AUTORES:}

Maria José de Oliveira Santos

Escola Superior de Saúde - Universidade de Trás-os-Montes e Alto Douro,Vila Real; Unidade de Investigação em Ciências da Saúde: Enfermagem / UICISA: E, da Escola Superior de Enfermagem de Coimbra;

mjsantos@utad.pt

Elisabete Soares Ferreira

Faculdade de Psicologia e Ciência da Educação da Universidade do Porto, Porto; elisabete@fpce.up.pt

Anabela Martins Pinto de Figueiredo

Escola Superior de Saúde - Universidade de Trás-os-Montes e Alto Douro, Vila Real afigueired@utad.pt

Manuela Maria da Conceição Ferreira

Escola Superior de Saúde de Viseu - Instituto Politécnico de Viseu; Viseu; Unidade de Investigação em Ciências da Saúde: Enfermagem / UICISA: E, da Escola Superior de Enfermagem de Coimbra; mmcferrreir@gmail.com 